Sir,

\section{Unilateral drug-induced ocular pseudopemphigoid}

Preservatives are an important component of ophthalmic preparations, providing antimicrobial activity and preventing decomposition of active drug. Often under recognized, however, are the significant cytotoxic effects of preservatives ${ }^{1}$ especially when associated with longterm therapy and with multidrug regimens as in chronic glaucoma.

An 85-year-old female patient presented in eye casualty with a painful red left eye and occasional discomfort of 2 months duration. She had been diagnosed with left pseudoexfoliative glaucoma 8 years earlier and was put on Xalatan, once at night (latanoprost $50 \mu \mathrm{g} / \mathrm{ml}$, Benzalkonium $0.02 \%$ ) and Iopidine, twice a day (apraclonodine $0.5 \%$, benzalkonium $0.01 \%$ ). Visual acuity was $6 / 12$ at this time. The patient then failed to attend for review for 2 years.

On presentation, at this time visual acuity had dropped to perception of light in the left eye. The history was not suggestive of use of any other topical treatment except that which was prescribed, and there was no element of physical and chemical injury.

Slit-lamp examination revealed that her right eye, which had not had any topical treatment, was normal, apart from significant lens opacities. The left eye showed signs of established keratoconjunctivitis sicca (but no clinical signs of this condition had been noted at earlier examinations) with cicatrization and keratinization of the conjunctiva and cornea. The conjunctival fornices were shallow and the cornea was opaque with active deep vascularization. Systemic examination revealed no skin or mucous membrane abnormalities.

Conjunctival biopsy was compatible with ocular cicatritial pemphigoid but negative for immunoflourescent staining for IgG, IgA, IgM, C3, and polyvalent antibodies.

Conjunctiva from the right eye was histologically normal. No circulating systemic antibodies were found.

In this case, it is likely that the preservative (benzalkonium chloride) in xalatan and Iopidine is the responsible agent causing ocular surface cicatrization and keratinization mimicking ocular pemphigoid.

When following up glaucoma patients, it is important to keep the possibility of drug-induced pseudopemphigoid in mind and the necessity to withdraw glaucoma treatment with preservatives. The toxic effects of benzalkonium chloride have been extensively reported and it may be necessary to consider use of preservative-free topical medications in certain glaucoma patients and an adjuvant of artificial tears, especially in the presence of early signs of pseudopemphigoid.

\section{References}

1 Neoker R. Effects of common ophthalmic preservatives on ocular health. Adv Ther 2001; 18(5): 205-215.

SK Gibran

Department of Ophthalmology

Cork University Hospital, Cork Ireland

Correspondence: SK Gibran

Tel: + 353214546400

Fax: + 353214922656

E-mail: syedgibran@yahoo.com

Eye (2004) 18, 1270. doi:10.1038/sj.eye.6701385

Published online 26 March 2004

Sir,

Distichiasis without lymphoedema?

Distichiasis arises from the aberrant differentiation of meibomian glands of the eyelid, resulting in the development of a few, or sometimes a second inner row, of 'eyelashes'. It can cause symptoms of corneal irritation and may need treatment. Primary distichiasis is thought to be only found as part of lymphoedema-distichiasis (LD) syndrome, although some individuals may only have signs of distichiasis and some only lymphoedema (less than 5\% in each case). ${ }^{1}$ Primary lymphoedema is an accumulation of interstitial fluid that occurs as a result of reduced lymph drainage. ${ }^{2}$ It is visible clinically as swelling of the affected part. We present a family with the condition, their genetic mutation, and discuss the ophthalmologist's role on encountering such a case.

\section{Case report}

A 28-year-old woman was seen in our eye clinic with symptomatic distichiasis. Severe irritation caused by aberrant eyelashes rubbing her corneae had made her seek treatment. Initially epilation and subsequently electrolysis of the offending eyelashes gave her relief. The remainder of the anterior and posterior segment examination was unremarkable. Interestingly, she was noted to have lymphoedema of both lower extremities (Figure 1). Physical examination revealed no other abnormalities.

\section{Family history}

Her two sons, aged 8 and 4 years, were also found to have distichiasis but both were asymptomatic (Figure 2). 


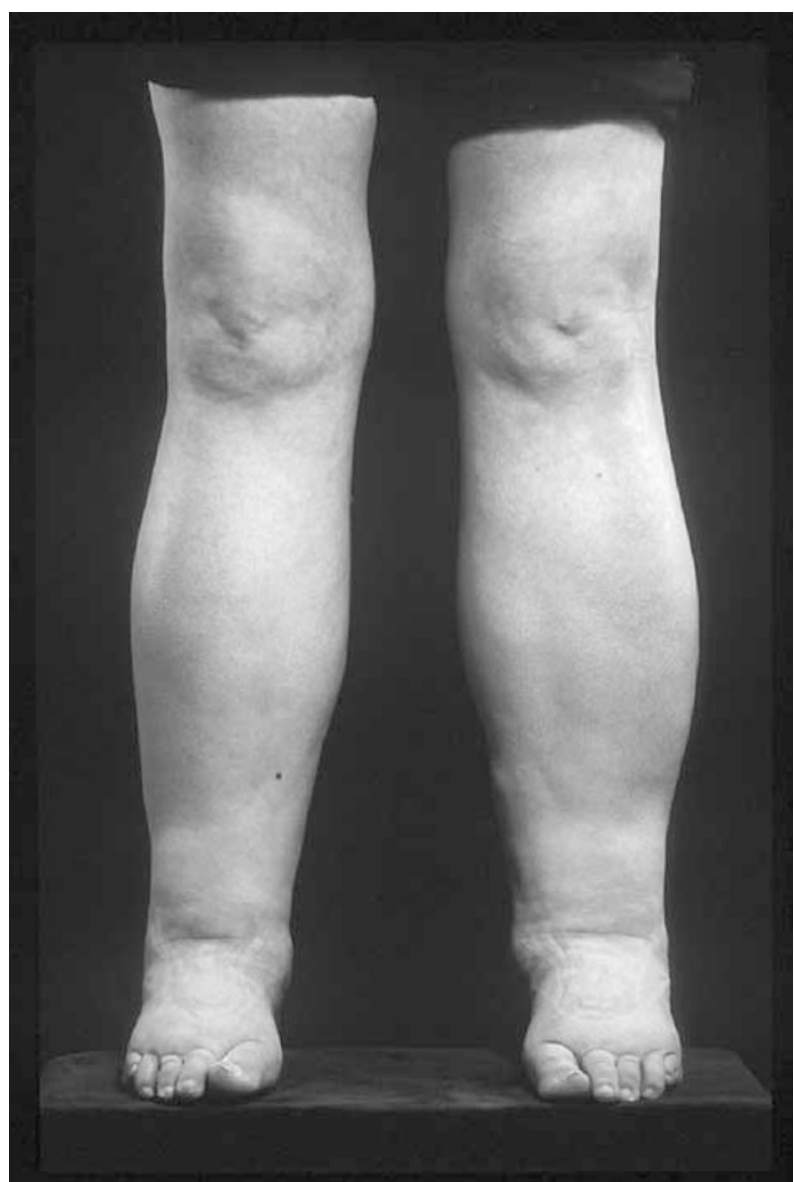

Figure 1 Lymphoedema of lower limbs of the proband.

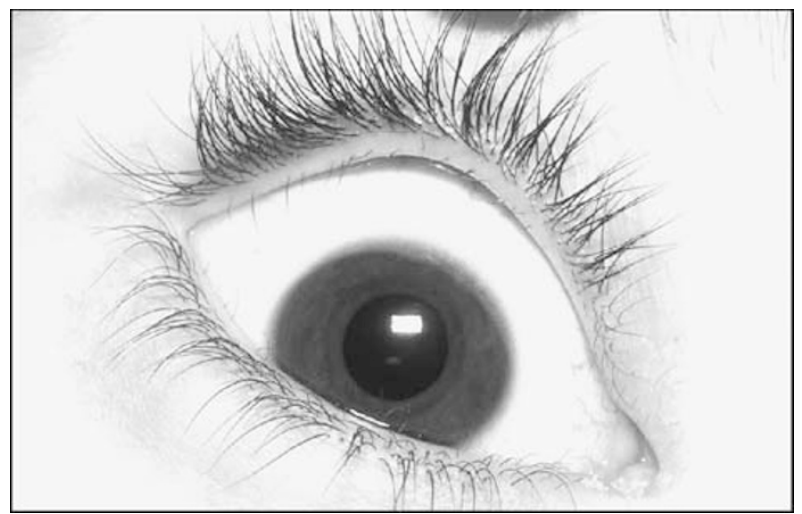

Figure 2 Distichiasis in 8-year-old son of the proband.

Their physical examinations were unremarkable with no lymphoedema. She had three brothers (two of them twins) with distichiasis, and a sister with distichiasis as well as lymphoedema. In addition, she had two unaffected siblings. As none of the unaffected siblings would subject themselves to examination and investigation, their genotype could not be ascertained.
A mutation in the FOXC2 gene was identified in the proband and shown to be inherited in both of her affected male offspring. This was a previously unpublished mutation, a 19 bp duplication: nt922923insGCGCTGCCCTACGCCGCCG.

\section{Discussion}

Congenital distichiasis may result when a primary epithelial germ cell destined to differentiate into a specialised sebaceous gland (meibomian gland) of the tarsus develops into a complete pilosebaceous unit. The condition is frequently inherited in an autosomal dominant pattern with high penetrance but variable expressivity. During infancy, these fine, misdirected cilia are usually well tolerated despite their apposition to the globe. Clinical presentation may be delayed until the age of 5 years, when patients may complain of chronic ocular irritation.

Acquired distichiasis, on the other hand, occurs in the setting of chronic inflammation, especially in cicatricial conditions like ocular pemphigoid, Stevens-Johnson syndrome and following chemical injuries of the eyelid. Unlike congenital distichiasis, the cilia of acquired distichiasis tend to be nonpigmented and stunted and are more likely to cause symptomatic ocular irritation. The two varieties are differentiated relatively easily.

LD syndrome is an autosomal dominant disorder that classically presents as lymphoedema of the lower limbs (age of onset variable, but most often after puberty) ${ }^{3}$ and aberrant eyelashes. Ocular associations reported with distichiasis are: corneal hypoaesthesia, ${ }^{4}$ photophobia, ptosis, congenital ectropion and entropion, congenital cataracts, exotropia; ${ }^{3}$ absent lacrimal duct; ${ }^{5}$ epicanthus, telecanthus, blepharophimosis, colour blindness, eyelid oedema; ${ }^{6}$ microphthalmos, dystrophic retinal pigmentation and optic disc pallor as in the oculocerebro-renal syndrome. ${ }^{7}$

Other features described include synophrys (meeting of the eyebrows in the midline), venous abnormalities; ${ }^{8}$ congenital heart defects; ${ }^{3}$ cleft palate; ${ }^{6}$ spinal tumours ${ }^{9}$ and ectrodactyly. ${ }^{10}$ More recently, generalised or localised iris abnormalities, small corneal diameter and opacities as well as optic nerve hypoplasia have been noted. ${ }^{11}$ None of these features were present in our family, although only a limited slit-lamp assessment was possible in the boys because of their age.

The clinical diagnosis of lymphoedema is not always straightforward except in advanced cases where classical skin changes and brawny swelling become obvious. The confirmatory investigation is lymphoscintigraphy but false negatives can occur, ${ }^{8}$ as can age-related false positives. In the report by Brice et al, ${ }^{12}$ males had an earlier onset of lymphoedema and a significantly 
increased risk of complications. The gene for $\mathrm{LD}$, located on the long arm of chromosome 16, is that encoding FOXC2, a forkhead family transcription factor involved in numerous developmental pathways. ${ }^{13}$ In all three members of the family presented in this report, a $19 \mathrm{bp}$ insertion in the FOXC2 gene was identified. To our knowledge this mutation has not been previously reported.

Distichiasis is the most consistently inherited feature of LD syndrome. Lymphoedema in this condition is highly penetrant; however, it is rarely recognised before puberty. While examining young children with distichiasis, the likelihood of them developing lymphoedema subsequently should be borne in mind and parents counselled accordingly. As there are many interesting and unanswered questions regarding the connection between various associated features in LD, further research may give us the answers. Every ophthalmologist, on recognising a case of distichiasis should take a clear family history asking about lymphoedema of the lower limbs and the other features listed above, and arrange molecular genetic investigations to look for mutations in the FOXC2 gene. The family should also be referred for genetic counselling as there can be associated cleft palate and/or congenital heart disease. Any families where distichiasis but no lymphoedema occur are also of great interest, as it is not clear whether mutations in the FOXC2 gene would account for this symptom alone.

\section{Acknowledgements}

P Mortimer, AH Child and S Mansour, along with RB, GB and SJ, are all part of the Lymphoedema Research Group at St George's Hospital Medical School, collaborating with M Sarfarazi, University of Connecticut Health Centre USA, which enables the lymphoedema research programme to continue. RB was funded by the British Heart Foundation. The genetic analysis was carried out within the network of the London IDEAS Genetic Knowledge Park.

\section{References}

1 Jeffrey S. How double eyelashes give you swollen legs. $\mathrm{Br} J$ Ophthalmol 2002; 86: 1074.

2 Witte MH. Genetic alterations in lymphedema. Lymphology 1997; 16: 19-25.

3 Erickson RP, Dagenais SL, Caulder MS, Downs CA, Herman $\mathrm{G}$, Jones MC et al. Clinical heterogeneity in lymphoedemadistichiasis with FOXC2 truncating mutations. J Med Genet 2001; 38(11): 761-766.

4 Kremer I, Weiberger D, Cohen S, Ben Sira I. Corneal hypoesthesia in asymptomatic familial distichiasis. $\mathrm{Br} \mathrm{J}$ Ophthalmol 1986; 70(2): 132-134.
5 Holder-Espinasse M, de Bluis MC, Faivrel L, Romana S, Uteza Y, Munnich A et al. Absent lacrimal ducts, distichiasis, dysmorphic features and brachydactyly: a case report. Clin Dysmorphol 2001; 10(4): 253-255.

6 O'Donnell BA, Collin JR. Distichiasis: Management with cryotherapy to the posterior lamella. Br J Ophthalmol 1993; 77(5): 289-292.

7 Brancato R, Matteini M, Cotrozzi G. Sindrome oculocerebro-renale in soggott portare di blefarophimosi e di distichiasis. Ann Ottal 1965; 91: 35-37.

8 Rosbotham JL, Brice GW, Child AH, Nunan TO, Mortimer PS, Burnand KG. Distichiasis-lymphoedema: clinical features, venous function \& lymphoscintigraphy. $\mathrm{Br}$ J Dermatol 2000; 142: 148-152.

9 Schwartz JF, O'Brien MS, Hoffman Jr JC. Hereditary spinal cysts, distichiasis and lymphedema. Ann Neurol 1980; 7: 340-343.

10 Lehmann OJ, Tuft S, Brice G, Smith R, Blixt A, Bell R et al. Novel anterior segment phenotypes resulting from forkhead gene alterations: evidence for cross-species conservation of function. Invest Ophthalmol Vis Sci 2003; 44: 2627-2633.

11 Collier MM. Ecrodactylie droite et distichiasis inferieur bilateral. Bull Soc D'Ophthalmol 1964; 64: 49-52.

12 Brice G, Mansour S, Bell R, Collin JR, Child AH, Brady AF et al. Analysis of the phenotypic abnormalities in lymphoedemadistichiasis syndrome in 74 patients with FOXC2 mutations or linkage to 16q24. J Med Genet 2002; 39(7): 478-483.

13 Fang J, Dagenais SL, Erickson RP, Arlt MF, Glynn MW, Gorski JL et al. Mutations in FOXC2 (MFH-1), forkhead family transcription factor, are responsible for the hereditary lyphedema-distichiasis syndrome. Am J Hum Genet 2000; 67: 1382-1388.

BB Patil ${ }^{1}$, R Bell², G Brice ${ }^{2}$, S Jeffery ${ }^{2}$ and SP Desai ${ }^{1}$ and the Lymphoedema Research Group ${ }^{2}$

\section{${ }^{1}$ Department of Ophthalmology \\ Doncaster Royal Infirmary, Doncaster UK \\ ${ }^{2}$ Medical Genetics Unit \\ St George's Medical School, London, UK}

Correspondence: SP Desai

Tel: + 441302366666 Ext: 3325

E-mail: desaisp@hotmail.com

Eye (2004) 18, 1270-1272. doi:10.1038/sj.eye.6701387

Published online 26 March 2004

Sir,

\section{CJD and intraocular surgery}

We read with interest the clinical study by Leslie et al, ${ }^{1}$ in the May (2003) issue of Eye. The authors eloquently showed the contamination results before and after the use of an automated rinsing system. They hypothesized that the contamination may be the cause of 